\title{
Leukocyte and Skeletal Muscle Telomere Length and Body Composition in Monozygotic Twin Pairs Discordant for Long-term Hormone Replacement Therapy
}

\author{
Elina Sillanpää, ${ }^{1, *}$ Paula Niskala, ${ }^{1}{ }^{*}$ Eija K. Laakkonen, ${ }^{1}$ Elodie Ponsot, ${ }^{2}$ Markku Alén, ${ }^{3,4}$ \\ Jaakko Kaprio, ${ }^{5,6,7}$ Fawzi Kadi, ${ }^{2}$ Vuokko Kovanen, ${ }^{1}$ and Sarianna Sipilä ${ }^{1}$ \\ ${ }^{1}$ Gerontology Research Centre, Faculty of Sport and Health Sciences, University of Jyväskylä, Jyväskylä, Finland \\ ${ }^{2}$ School of Health and Medical Sciences, Örebro University, Örebro, Sweden \\ ${ }^{3}$ Department of Medical Rehabilitation, Oulu University Hospital, Oulu, Finland \\ ${ }^{4}$ Center for Life Course Health Research, University of Oulu, Oulu, Finland \\ ${ }^{5}$ Department of Public Health, University of Helsinki, Helsinki, Finland \\ ${ }^{6}$ Institute for Molecular Medicine (FIMM), University of Helsinki, Helsinki, Finland \\ ${ }^{7}$ National Institute for Health and Welfare, Helsinki, Finland
}

\begin{abstract}
Estrogen-based hormone replacement therapy (HRT) may be associated with deceleration of cellular aging. We investigated whether long-term HRT has effects on leukocyte (LTL) or mean and minimum skeletal muscle telomere length (SMTL) in a design that controls for genotype and childhood environment. Associations between telomeres, body composition, and physical performance were also examined. Eleven monozygotic twin pairs (age $57.6 \pm 1.8$ years) discordant for HRT were studied. Mean duration of HRT use was $7.3 \pm 3.7$ years in the user sister, while their co-twins had never used HRT. LTL was measured by qPCR and SMTLs by southern blot. Body and muscle composition were estimated by bioimpedance and computed tomography, respectively. Physical performance was measured by jumping height and grip strength. HRT users and non-users did not differ in LTL or mean or minimum SMTL. Within-pair correlations were high in LTL $(r=0.69, p=.020)$ and in mean $(r=0.74, p=.014)$ and minimum SMTL $(r=0.88, p=.001)$. Body composition and performance were better in users than non-users. In analyses of individuals, LTL was associated with BMI $\left(r^{2}=0.30, p=.030\right)$, percentage total body $\left(r^{2}=0.43, p=.014\right)$, and thigh $\left(r^{2}=\right.$ $0.55, p=.004)$ fat, while minimum SMTL was associated with fat-free mass $\left(r^{2}=0.27, p=.020\right)$ and thigh muscle area $\left(r^{2}=0.42, p=.016\right)$. We found no associations between HRT use and telomere length. Longer LTLs were associated with lower total and regional fat, while longer minimum SMTLs were associated with higher fat-free mass and greater thigh muscle area. This suggests that telomeres measured from different tissues may have different associations with measures of body composition.
\end{abstract}

Keywords: estrogen, telomeres, percentage of fat, fat-free mass, computed tomography, bioimpedance, twin design, post-menopausal

Telomeres, which are non-coding DNA sequences capping the end of eukaryotic chromosomes, are known to play an important role in genome protection. In cell cultures, telomeres have been shown to shorten after each round of cell division (Allsopp et al., 1992) until they reach a critical length and the cell loses its ability to divide further. In this respect, telomere length has been used as an indicator of both replicative history and regenerative potential. Telomere erosion can also be counterbalanced by telomerase, an enzyme able to elongate telomeric ends, such as in highly
ReCeIved 18 August 2016; ACCEPTEd 5 December 2016. First published online 14 February 2017.

ADDRESS FOR CORRESPONDENCE: Elina Sillanpää, PhD, Docent, Gerontology Research Center, Faculty of Sport and Health Sciences, University of Jyväskylä, P.O. Box 35 (VIV), FIN-40014 Jyväskylä, Finland. E-mail: elina.sillanpaa@jyu.fi

* These authors contributed equally to this work. 
proliferating stem cells (Blasco, 2005; Bodnar et al., 1998; Oeseburg et al., 2010).

Aging is known to be associated with telomere shortening in some tissues, such as blood, whereas in other tissues, such as skeletal muscle, the question is still a matter of debate (Daniali et al., 2013; Decary et al., 1997; Ponsot et al., 2008). The in vivo biology of telomeres remains imperfectly understood and may be governed by several other regulatory factors (de Lange, 2006; Ponsot et al., 2012). Such complex interplay could partly explain why telomere length is not influenced by aging alone but also by environmental factors. For example, chronic life stress has been suggested to accelerate blood telomere shortening (Epel et al., 2004), whereas physical training has been associated with longer telomeres in leukocytes (Cherkas et al., 2008; Du et al., 2012; LaRocca et al., 2010) and in skeletal muscle (Kadi et al., 2008).

Telomere length does not vary between the sexes at birth; however, during adulthood, women consistently exhibit longer telomeres in leukocytes (Aviv et al., 2005; Benetos et al., 2001; Nordfjall, Eliasson, Stegmayr, Melander et al., 2008). Estrogens may protect telomeres from oxidative stress and DNA damage by decreasing the amount of reactive oxygen species and influencing vascular biology (Aviv, 2002; Song et al., 2009; Wong et al., 2008), stimulating telomerase (Grasselli et al., 2008; Kyo et al., 1999; Misiti et al., 2000), and affecting telomerase activation through other indirect mechanisms (Rahimian et al., 2004; Vasa et al., 2000). Earlier studies have shown that longer exposure to endogenous estrogen, that is, longer duration of the reproductive years, is associated with longer telomere length in leukocytes (Gray et al., 2014; Lee et al., 2005), peripheral mononuclear cells (Lin et al., 2011), and primordial germ cells (Aydos et al., 2005), and inversely associated with telomerase activity (Lin et al., 2011). Estrogen metabolism has also been suggested to be associated with telomere length through mechanisms linked to changes in body composition. After menopause, fat mass and, in particular, central obesity is increased, expressed as elevated insulin resistance and leptin levels (Aviv et al., 2006). Fat tissue is a source of leptin and adiponectin, both of which affect inflammation and insulin resistance. Inflammation enhances the turnover rate of leukocytes, and oxidative stress enhances the loss of telomere repeat per cell replication.

It is reasonable to postulate that estrogen metabolism is related to telomere length and/or attrition rate by affecting the number of cell divisions and by protecting telomere sequences. Endogenous estrogens may, however, have different effects on telomeres than the exogenous estrogens (Lin et al., 2011) that are used in hormone replacement therapy (HRT). The couple of studies done so far have reported inconsistent results. Lin et al. (2011) found no association between longer duration of HRT and either leukocyte telomere length (LTL) or telomerase activity, whereas Lee et al. (2005) reported longer LTL in long-term HRT users compared to women who had never used HRT. In theory, HRT may have direct effects on telomeres, but it can also act as an anti-aging agent by maintaining healthier body composition and physical performance (Ronkainen et al., 2009). We have shown previously that HRT promotes healthier inflammatory profile (Kangas et al., 2014), and amount and distribution of body fat as well as better physical performance (Ronkainen et al., 2009). Earlier studies have suggested that healthier body composition might protect telomere ends from accelerated shortening (Lee et al., 2011; Njajou et al., 2012; Valdes et al., 2005).

Telomere length varies between individuals and between different cells and tissues within the same individual. Leukocytes represent a constantly dividing cell population that presumably should show early signs of wear and tear as short telomeres. Adult muscle cells on the other hand are multinucleated post-mitotic cells that are not considered to divide and thereby consume telomeres after differentiation, but rather to describe an inherited telomere length. However, skeletal muscles also contain a population of quiescent muscle stem cells, called satellite cells, which can reenter the cell cycle to initiate proliferation and donate nuclei to muscle fibers. Satellite cells are essential for muscle regeneration and are responsible for muscle growth (Alway et al., 2014). A significant inverse relationship between age and skeletal muscle telomere length (SMTL) has been reported across the age span (Decary et al., 1997; Wernig et al., 2005), potentially limiting the proliferative capacity of satellite cells. That may lead to satellite cell senescence, thereby contributing to a loss of skeletal muscle mass and consequently physical performance.

Theoretically, better preserved telomere length in satellite cells may partly explain the previously reported positive association between HRT and musculature (Pollanen et al., 2007; Ronkainen et al., 2009; Sipila et al., 2001). We hypothesized that HRT use protects telomeres from aging-associated shortening and that telomere attrition rate may be partially genetically determined. We used a design that controls for genotype and childhood environment (monozygotic twin pairs discordant for HRT) to investigate the possible association of long-term HRT use on telomere length in leukocytes and in skeletal muscle in order to define possible difference in protection of telomere length in mitotic (defined as relative LTL) and post-mitotic (defined as mean SMTL, SMTL, and minimum SMTL, which is assumed to represent satellite cell telomere length) cell lineages due to HRT. In addition, associations between LTL, mean, or minimum SMTL, and total body and thigh composition and physical performance were examined.

\section{Material and Methods}

\section{Study Design}

This study is part of a larger study, 'Sarcopenia - Skeletal Muscle Adaptation to Postmenopausal Hypogonadism 
and Effects of Hormone Replacement Therapy and Physical Activity in Older Women: A Genetic and Molecular Biological Study on Estrogen-related Pathways' (SAWEs). The recruitment process has been described in detail elsewhere (Ronkainen et al., 2009). Briefly, 537 monozygotic (MZ) female twin pairs from selected age groups of the population-based Finnish Twin Cohort (Kaprio \& Koskenvuo, 2002) were invited to self-select themselves as discordant for the use of HRT. A total of $15 \mathrm{MZ}$ pairs who responded to the invitation met the inclusion criteria and had no contraindications for participation in the measurements and muscle biopsy sampling, and were invited to the laboratory examinations. Four of these twin pairs were then excluded from the present analysis owing to the use of tibolone, which is not an estrogen-based HRT treatment. Zygosity was verified at the paternity testing laboratory (National Public Health Institute, Helsinki, Finland) using DNA extracted from a venous blood sample with a battery of 10 highly polymorphic gene markers. All laboratory measurements and data analyses were carried out blind to HRT status.

Mean duration of HRT among the users was 7.3 (SD 3.7 , range 2-16) years, while their co-twins had never used HRT. Of the HRT users, five women used estradiol-only (1-2 mg) preparations and six a combined treatment comprising both estrogenic (1-2 mg) and progestogenic compounds. We have reported earlier that while the HRT users had higher levels of both total and free $17 \beta$-estradiol $\left(E_{2}\right)$ and total estrone $\left(\mathrm{E}_{1}\right)$ than their non-user sisters, both cotwins were similar in their physical activity (PA) habits, daily energy intake, use of medication, and smoking behavior (Ronkainen et al., 2009; Ronkainen et al., 2010).

The Ethics Committee of the Central Finland Health Care District approved the study and it was conducted according to the guidelines laid down by the World Medical Association in the Declaration of Helsinki (2000). Written informed consent was provided by the participants before the measurements.

\section{Telomere Length Measurements}

Relative LTL was determined from peripheral blood DNA by a quantitative real-time polymerase chain reaction (qPCR) based method, originally developed by Cawthon et al. (2003), with minor modifications (Eerola et al., 2010; Kananen et al., 2010). Briefly, a separate qPCR reaction was performed with telomere sequence-specific primers and a single copy control gene, $\beta$-hemoglobin. Both the telomere and $\beta$-hemoglobin reactions were performed with a CFX 384 Real-Time PCR Detection System (Bio-Rad, Hercules, CA). Melt-curve analysis was carried out to ensure specific primer binding. A DNA dilution series $(0.5,1.0,2.0$, $5.0,10,20$, and $30 \mathrm{ng}$ ) was used to create a standard curve $\left(R^{2}=0.997\right.$ for the telomere and 0.998 for the $\beta$ hemoglobin run) and to perform absolute quantification of each sample. We used Bio-Rad CFX Manager v.1.6 software to perform quality control and to calculate the T/S (telomere to single-copy gene intensity) ratios for the samples to obtain the relative telomere length. The correlation coefficient of variation $(\mathrm{CV})$ for repeat measures was $6.37 \%$ for the telomere reaction, $4.99 \%$ for the $\beta$-hemoglobin reaction, and $6.97 \%$ for the ratio (T/S), as determined by running six control samples across six plates.

Mean and minimum SMTL was determined from a muscle biopsy sample obtained from the mid part of the vastus lateralis, defined as the midpoint between the greater trochanter and the lateral joint line of the knee. Following the removal of all visible residues of fat and connective tissue, the biopsy samples were frozen in liquid nitrogen and stored at $-80^{\circ} \mathrm{C}$ until use. A southern blot protocol specifically adapted for the study of skeletal muscle was used in the analysis (Decary et al., 1997; Ponsot et al., 2008). Total genomic DNA was digested with the restriction enzyme HinfI (New England Biolabs, Ipswich, MA, USA), and telomeric terminal restriction fragments were generated. Digested DNA, together with two DNA ladders ( $1 \mathrm{~kb}$ plus and high molecular weight; Invitrogen Life technologies, Paisley, UK), were resolved using electrophoresis in $0.7 \%$ agarose gels. The telomeric terminal restriction fragments were detected by hybridization to a ${ }^{32} \mathrm{P}$-labelled (TTAGGG) $)_{4}$. The signals were analyzed using a computerassisted system (Scion image 4.03 software; Scion Corporation, Bethesda, MD, USA) and modeled using an improved signal analysis model for the assessment of telomere restriction fragment length (Ponsot et al., 2008). Mean SMTL comprises telomeres from all cells, of which the majority contain post-mitotic myonuclei, which prior to differentiation into muscle fiber cells had undergone only a few mitoses. In contrast, minimum SMTL shows the length of the shortest telomeres in the sample. Hypothetically, the shortest telomeres are found in satellite cells and in myonuclei that have been added to the myofibers during the most recent mitotic cycles (Ponsot et al., 2008). SMTL was successfully determined in 10 twin pairs. The $\mathrm{CV}$ of telomere length measurements in skeletal muscle has been below 5\% for both mean and minimum length (Ponsot \& Kadi, 2008).

\section{Total Body and Thigh Composition}

Body mass index was calculated as a function of measured body weight and height $\left(\mathrm{kg} / \mathrm{m}^{2}\right)$. Percentage body fat and total body fat-free mass (FFM) were measured by a multifrequency bioelectrical impedance method (InBody [720], Biospace, Seoul, Korea) after overnight fast. In our laboratory, the CV for two consecutive measurements of percentage of body fat has been $0.6 \%$.

Thigh composition was assessed from computed tomography scans (Siemens Somatom Emotion scanner, Siemens AG, Erlangen, Germany) obtained from the same site as the muscle biopsy. Thigh muscle compartment cross-sectional area, thigh fat areas (total fat area, subcutaneous fat, and fat infiltrated into muscle tissue), and relative proportion of fat 
(\%) were analyzed using software developed at the University of Jyväskylä for cross-sectional computed tomography image analysis (Geanie 2.1, Commit; Ltd., Espoo, Finland), as described earlier (Ronkainen et al., 2009). In our laboratory, the CV between two consecutive measurements was 1 to $2 \%$ for muscle area (Sipila et al., 2001).

\section{Physical Performance}

Vertical jumping height $(\mathrm{cm})$ was calculated from flight time during a countermovement jump and measured on a contact mat (Bosco et al., 1983). Three maximal efforts were conducted and the best result was used in the analysis. In our laboratory, the CV between two consecutive measurements has been $5 \%$ (Sipila et al., 2001). Vertical jumping height can be used as an estimate of lower body muscle power, that is, the ability of the neuromuscular system to produce the greatest possible force as rapidly as possible.

Hand grip strength $(\mathrm{kg})$ was measured using an isometric dynamometer (Good Strength IGS01, Metitur Oy, Jyväskylä, Finland). Subjects were encouraged verbally to produce maximal contraction. Hand grip strength was recorded in kilograms to within accuracy of $0.1 \mathrm{~kg}$ and the best attempt of three trials was used in the analysis.

\section{Physical Activity}

PA was assessed using a modified version of the seven-point Grimby scale. The categories used in questionnaire were (1) inactive, (2) light activity 1-2 times per week, (3) light activity several times per week, (4) moderate activity 1-2 times per week, (5) moderate activity several times per week, (6) high activity several times per week, and (7) competitive sports several times per week. For the analysis, categories 1 and 2, 3 and 4, and 5 to 7 were combined to describe inactive, moderate, and vigorous activity groups.

\section{Statistics}

Owing to the relatively small number of observations, the statistical significance of the differences between the means of the HRT users and non-users was tested with Wilcoxon's signed rank test. Data are shown as means and standard deviations, unless otherwise stated. Associations between age and leukocyte and skeletal muscle telomeres were analyzed using standardized regression coefficients to represent bivariate correlations, and $p$ values were adjusted for twin dependency. Associations between telomere length and body composition, and telomere length and physical performance were tested with linear regression analysis. In regression models, within-pair dependency of twin individuals was taken into account using the cluster option in analysis. Possible confounders - age, level of PA, and HRT (user or non-user) - were entered into the model one at a time. Intraclass correlation coefficients (ICC) were computed for the twin pairs to estimate the level of within-pair similarity. The level of significance was set at $p \leq .05$. Data analyses were carried out with IBM SPSS Statistics 22 (SPSS
Inc., Chicago, IL) and Stata version 14 (Stata Corp, College Station, TX, USA).

\section{Results}

Participants' Characteristics and Telomere Length

The mean age of the participants was 57.6 (SD 1.8, range, 55-62 years). Mean LTL, mean SMTL, and minimum SMTL did not differ between the HRT users and their cotwins (Table 1). On average, the HRT users had lower percentage body fat than non-user sisters, while no differences between the groups were observed in FFM. Moreover, no differences were observed between HRT users and nonusers in thigh muscle area or amount of fat infiltrated into the muscle compartment. However, thigh fat area, relative proportion of fat, and subcutaneous fat were smaller in users than non-users. HRT users had better vertical jumping height, indicating higher muscle power, than their cotwins, but no difference was observed in hand grip strength.

\section{Associations Between Leukocyte and Skeletal Muscle Telomere Length and Age}

No significant relationship was found between LTL and mean $(r=0.114, p=.12)$ and minimum SMTL $(r=0.172$, $p=.47)$. Mean and minimum SMTLs were not associated $(r=0.220, p=.38)$. Age was not associated with LTL $(r=-0.070, p=.69)$, minimum SMTL $(r=-0.43, p=.12)$, or mean SMTL $(r=-0.40, p=.097)$.

Within-pair analysis showed that LTL of HRT users correlated significantly with the corresponding values of nonusers ( $r=0.69, p=.020$, Figure $1 \mathrm{~A})$. Pairwise correlations were even higher in twin pairs for mean $(r=0.74, p=.014)$ and minimum $(r=0.88, p=.001)$ SMTL; see Figure $1 \mathrm{~A}, \mathrm{~B}$, and $\mathrm{C}$.

Associations Between Leukocyte Telomere Length (LTL), Total Body and Thigh Composition, and Physical Performance

In all participants, longer LTL was associated with lower $\mathrm{BMI}$, percentage body fat, and relative proportion of fat in the thigh (Table 2, Figure 2A and B). Borderline significant association between longer LTL and lower thigh fat area, subcutaneous fat, and infiltrated fat were observed. Significant associations were not observed between LTL and FFM or physical performance. Linear regression revealed that BMI, percentage body fat, thigh fat area, relative proportion of fat, and subcutaneous fat were significant independent predictors of LTL after adjustment for age, PA level, and HRT use.

Associations Between Mean and Minimum Skeletal Muscle Telomere Length, Total Body and Thigh Composition and Physical Performance

Mean SMTL was not associated with total body composition or vertical jumping height (Table 3). A borderline significant positive association was found between mean 


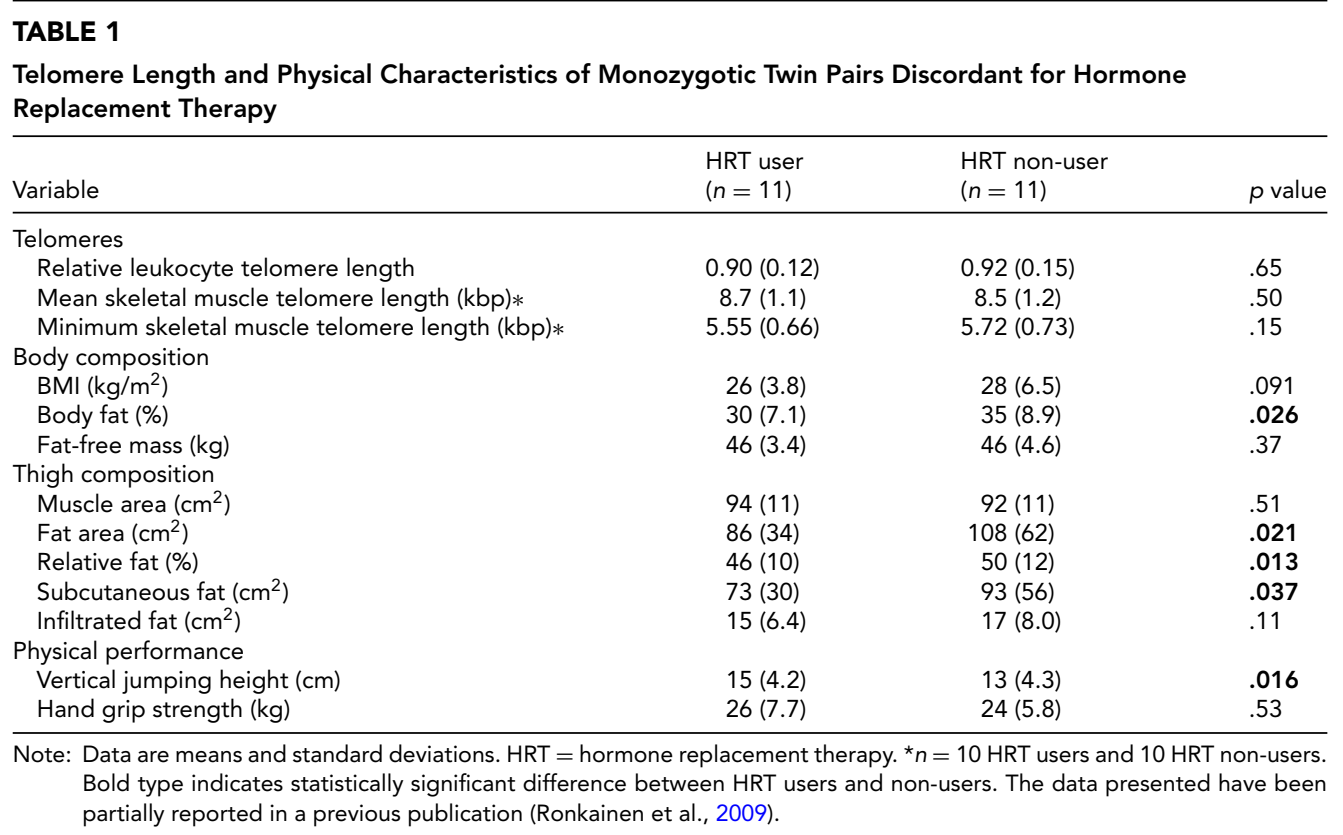

SMTL and grip strength. Participants with larger total body FFM and greater thigh muscle area had longer minimum SMTL, but measures of total body or thigh fat or physical performance were not associated with SMTL (Table 4, Figures 3A and B). Regression models showed that FFM and thigh muscle area were significant independent predictors of minimum SMTL after adjusting for age, PA level, and HRT use.

\section{Discussion}

The results showed that the use of the long-term, estrogenbased HRT was not significantly associated with either leukocyte or SMTL. This finding is strengthened by the observation that pairwise correlations in LTL and SMTL between the MZ twin sisters discordant for HRT were high, despite the HRT use and more favorable total and regional body composition and better muscle power observed in the HRT users (Ronkainen et al., 2009). Our results also revealed that longer telomere lengths were associated with healthier body composition and less clearly with physical performance. LTL, a biomarker that has been associated with inflammation, stress reaction, cardiovascular diseases, and mortality (Cawthon et al., 2003; Demissie et al., 2006; Epel et al., 2004; Haycock et al., 2014; Huzen et al., 2010) was related to lower measures of total body fat and thigh fat compartments. In contrast, the shortest telomeres present in the vastus lateralis muscle tissue, that is minimum SMTLs, were associated with total body FFM and thigh muscle area, but not body fatness.

A discordant twin study is a particularly suitable design for investigating the environmental exposures such as HRT during late adulthood, since it allows controlling for genetic factors and also most of the environmental exposures that occur during the first years of life. This is especially important with regard to telomeres, as it is known that telomere length is highly heritable (Broer et al., 2013; Hjelmborg et al., 2015) and the majority of the attrition occurs during the first two decades, when cells divide and differentiate rapidly due to growth and development (Aubert et al., 2012; Zeichner et al., 1999). Our results from a genetically controlled sample suggest that long-term HRT is not associated with telomere length in older age. There are several possible explanations for that observation. First, it might be that exogenous estrogens (HRT) do not have a similar protective effect on telomere attrition as endogenous estrogens. Second, it is possible and highly likely, based on ours and others' findings, that telomere length and possibly also attrition rate are strongly genetically regulated. It might be that similar genetic factors regulate both natural hormonal aging and telomere length, and therefore, for example, menopause occurs at later age in subjects with longer telomeres and longer life expectancy. Third, it might be, that telomere attrition during late adulthood is so minor that even the effect of the average use of HRT use over 7 years is beyond our measurement limit (Chen et al., 2011).

Our result is in line with findings of Lin et al. (2011), who reported no associations between HRT and peripheral mononuclear telomere length or telomerase activity, and Dalgård et al. (2015), who investigated leukocyte telomeres. However, Lee et al. (2005) reported higher telomere length in postmenopausal HRT users compared to nonusers. The disparity between our results and those of Lee et al. might be related to selection bias. In the study by Lee et al., HRT users participated more often in regular exercise and used more vitamin supplements than nonusers. In the present sample, not only genotype, but also PA habits, daily energy intake, use of medication, and smoking 
TABLE 2

Associations between Relative Leukocyte Telomere Length and Estimates of Total Body and Thigh Composition and Physical Performance in All Subjects $(n=22)$

\begin{tabular}{|c|c|c|c|c|c|}
\hline & \multicolumn{3}{|c|}{ Relative leukocyte telomere length } & \multicolumn{2}{|c|}{ Model* } \\
\hline & $\beta$ & SE & $p$ & $r^{2}$ & $p$ \\
\hline \multicolumn{6}{|l|}{ Body composition } \\
\hline \multicolumn{6}{|l|}{ Body mass index $\left(\mathrm{kg} / \mathrm{m}^{2}\right)$} \\
\hline Model 1 & -0.014 & 0.005 & .030 & 0.302 & .030 \\
\hline Model 2: adjusted with age & -0.015 & 0.005 & .017 & 0.342 & .009 \\
\hline Model 3: as 2 and PA & -0.015 & 0.005 & .013 & 0.385 & .075 \\
\hline Model 4: as 3 and HRT & -0.016 & 0.004 & .004 & 0.410 & .031 \\
\hline \multicolumn{6}{|l|}{ Percentage body fat (\%) } \\
\hline Model 1 & -0.011 & 0.004 & .014 & 0.434 & .014 \\
\hline Model 2: adjusted with age & -0.011 & 0.004 & .010 & 0.468 & .002 \\
\hline Model 3: as 2 and PA & -0.011 & 0.003 & .006 & 0.526 & .026 \\
\hline Model 4: as 3 and HRT & -0.012 & 0.003 & .002 & 0.569 & .020 \\
\hline \multicolumn{6}{|l|}{ Fat-free mass $(\mathrm{kg})$} \\
\hline Model 1 & -0.006 & 0.007 & .397 & 0.030 & .397 \\
\hline Model 2: adjusted with age & -0.007 & 0.006 & .299 & 0.045 & .569 \\
\hline Model 3: as 2 and PA & -0.007 & 0.007 & .296 & 0.109 & .498 \\
\hline Model 4: as 3 and HRT & -0.007 & 0.007 & .296 & 0.109 & .694 \\
\hline \multicolumn{6}{|l|}{ Thigh composition } \\
\hline \multicolumn{6}{|l|}{ Muscle area $\left(\mathrm{m}^{2}\right)$} \\
\hline Model 1 & 0.102 & 0.287 & .729 & 0.007 & .729 \\
\hline Model 2: adjusted with age & 0.097 & 0.301 & .755 & 0.011 & .813 \\
\hline Model 3: as 2 and PA & 0.126 & 0.311 & .694 & 0.075 & .394 \\
\hline Model 4: as 3 and HRT & 0.126 & 0.310 & .692 & 0.075 & .587 \\
\hline \multicolumn{6}{|l|}{ Fat area $\left(\mathrm{m}^{2}\right)$} \\
\hline Model 1 & -0.146 & 0.074 & .076 & 0.298 & .076 \\
\hline Model 2: adjusted with age & -0.164 & 0.075 & .053 & 0.350 & .071 \\
\hline Model 3: as 2 and PA & -0.157 & 0.071 & .051 & 0.370 & .124 \\
\hline Model 4: as 3 and HRT & -0.171 & 0.069 & .033 & 0.398 & .196 \\
\hline \multicolumn{6}{|l|}{ Relative fat $(\%)$} \\
\hline Model 1 & -0.009 & 0.002 & .004 & 0.547 & .004 \\
\hline Model 2: adjusted with age & -0.009 & 0.002 & .002 & 0.601 & .008 \\
\hline Model 3: as 2 and PA & -0.009 & 0.002 & .002 & 0.609 & .007 \\
\hline Model 4: as 3 and HRT & -0.010 & 0.002 & .001 & 0.650 & .013 \\
\hline \multicolumn{6}{|l|}{ Subcutaneous fat $\left(\mathrm{m}^{2}\right)$} \\
\hline Model 1 & -0.155 & 0.079 & .078 & 0.272 & .078 \\
\hline Model 2: adjusted with age & -0.173 & 0.080 & .056 & 0.319 & .103 \\
\hline Model 3: as 2 and PA & -0.164 & 0.076 & .057 & 0.336 & .150 \\
\hline Model 4: as 3 and HRT & -0.180 & 0.074 & .036 & 0.362 & .221 \\
\hline \multicolumn{6}{|l|}{ Infiltrated fat $\left(\mathrm{m}^{2}\right)$} \\
\hline Model 1 & -0.911 & 0.472 & .082 & 0.235 & .082 \\
\hline Model 2: adjusted with age & -0.952 & 0.466 & .068 & 0.256 & .178 \\
\hline Model 3: as 2 and PA & -0.993 & 0.453 & .053 & 0.335 & .301 \\
\hline Model 4: as 3 and HRT & -1.011 & 0.474 & .058 & 0.340 & .385 \\
\hline \multicolumn{6}{|l|}{ Physical performance } \\
\hline \multicolumn{6}{|l|}{ Vertical jumping height $(\mathrm{cm})$} \\
\hline Model 1 & 0.013 & 0.009 & .173 & 0.181 & .173 \\
\hline Model 2: adjusted with age & 0.014 & 0.009 & .140 & 0.205 & .156 \\
\hline Model 3: as 2 and PA & 0.016 & 0.009 & .101 & 0.302 & .179 \\
\hline Model 4: as 3 and HRT & 0.017 & 0.009 & .104 & 0.314 & .306 \\
\hline \multicolumn{6}{|l|}{ Hand grip strength $(\mathrm{kg})$} \\
\hline Model 1 & 0.006 & 0.005 & .261 & 0.105 & .261 \\
\hline Model 2: adjusted with age & 0.007 & 0.001 & .244 & 0.130 & .519 \\
\hline Model 3: as 2 and PA & 0.011 & 0.005 & .066 & 0.304 & .258 \\
\hline Model 4: as 3 and HRT & 0.011 & 0.005 & .066 & 0.416 & .304 \\
\hline
\end{tabular}

behavior were similar among the twin pairs discordant for HRT (Ronkainen et al., 2009; Ronkainen et al., 2010). However, HRT users have been shown to have better mobility, greater muscle power, and more favorable body and muscle composition than non-users (Ronkainen et al., 2009).

The novel finding of this study was that in MZ twin sisters, both mean and minimum SMTL showed a high correlation, indicating strong familiality, possibly due to major genetic effects. As expected, the correlation between twin sisters was higher in post-mitotic SMTL compared to length of the telomeres in more rapidly dividing leukocytes. However, a higher correlation was observed in minimum SMTL compared to LTL and mean SMTL, which suggest that telomere attrition rate may also be genetically regulated. So far, very few papers have compared telomeres measured in leukocytes and those in skeletal muscle cells. 
TABLE 3

Associations Between Mean Skeletal Muscle Telomere Length and Estimates of Total Body and Thigh Composition and Physical Performance in All Subjects $(n=20)$

\begin{tabular}{|c|c|c|c|c|c|}
\hline & \multicolumn{3}{|c|}{ Skeletal muscle mean telomere length } & \multicolumn{2}{|c|}{ Model* } \\
\hline & $\beta$ & SE & $p$ & $r^{2}$ & $p$ \\
\hline \multicolumn{6}{|l|}{ Body composition } \\
\hline \multicolumn{6}{|l|}{ Body mass index $\left(\mathrm{kg} / \mathrm{m}^{2}\right)$} \\
\hline Model 1 & 0.007 & 0.050 & .893 & 0.000 & .893 \\
\hline Model 2: adjusted with age & -0.072 & 0.060 & .261 & 0.193 & .123 \\
\hline Model 3: as 2 and PA & -0.065 & 0.063 & .327 & 0.202 & .015 \\
\hline Model 4: as 3 and HRT & -0.066 & 0.071 & .376 & 0.202 & .037 \\
\hline \multicolumn{6}{|l|}{ Percentage body fat (\%) } \\
\hline Model 1 & 0.031 & 0.037 & .429 & 0.016 & .429 \\
\hline Model 2: adjusted with age & -0.006 & 0.046 & .898 & 0.162 & .264 \\
\hline Model 3: as 2 and PA & -0.003 & 0.047 & .956 & 0.177 & .018 \\
\hline Model 4: as 3 and HRT & 0.003 & 0.048 & .955 & 0.180 & .053 \\
\hline \multicolumn{6}{|l|}{ Fat-free mass $(\mathrm{kg})$} \\
\hline Model 1 & 0.072 & 0.167 & .677 & 0.021 & .677 \\
\hline Model 2: adjusted with age & -0.037 & 0.195 & .854 & 0.224 & .166 \\
\hline Model 3: as 2 and PA & -0.033 & 0.198 & .871 & 0.180 & .002 \\
\hline Model 4: as 3 and HRT & -0.032 & 0.201 & .878 & 0.183 & .008 \\
\hline \multirow{2}{*}{\multicolumn{6}{|c|}{$\begin{array}{l}\text { Thigh composition } \\
\text { Muscle area }\left(\mathrm{m}^{2}\right)\end{array}$}} \\
\hline & & & & & \\
\hline Model 1 & -2.076 & 2.596 & .445 & 0.015 & .445 \\
\hline Model 2: adjusted with age & -3.641 & 3.450 & .319 & 0.205 & .098 \\
\hline Model 3: as 2 and PA & -3.363 & 3.400 & .348 & 0.213 & .026 \\
\hline Model 4: as 3 and HRT & -3.393 & 3.363 & .339 & 0.216 & .071 \\
\hline \multicolumn{6}{|l|}{ Fat area $\left(m^{2}\right)$} \\
\hline Model 1 & -0.542 & 0.381 & .189 & 0.024 & .189 \\
\hline Model 2: adjusted with age & -1.248 & 0.504 & .035 & 0.270 & .035 \\
\hline Model 3: as 2 and PA & -1.206 & 0.531 & .049 & 0.272 & .011 \\
\hline Model 4: as 3 and HRT & -1.286 & 0.629 & .071 & 0.275 & .029 \\
\hline \multicolumn{6}{|l|}{ Relative fat (\%) } \\
\hline Model 1 & -0.013 & 0.014 & .375 & 0.007 & .375 \\
\hline Model 2: adjusted with age & -0.034 & 0.023 & .163 & 0.208 & .119 \\
\hline Model 3: as 2 and PA & -0.031 & 0.025 & .235 & 0.213 & .015 \\
\hline Model 4: as 3 and HRT & -0.031 & 0.026 & .251 & .213 & .035 \\
\hline \multicolumn{6}{|l|}{ Subcutaneous fat $\left(\mathrm{m}^{2}\right)$} \\
\hline Model 1 & -0.729 & 0.505 & .183 & 0.036 & .183 \\
\hline Model 2: adjusted with age & -1.454 & 0.562 & .029 & 0.286 & .020 \\
\hline Model 3: as 2 and PA & -1.420 & 0.581 & .037 & 0.287 & .009 \\
\hline Model 4: as 3 and HRT & -1.524 & 0.697 & .057 & 0.291 & .027 \\
\hline \multicolumn{6}{|l|}{ Infiltrated fat $\left(\mathrm{m}^{2}\right)$} \\
\hline Model 1 & 2.125 & 4.747 & .665 & 0.007 & .665 \\
\hline Model 2: adjusted with age & -1.067 & 5.029 & .837 & 0.163 & .260 \\
\hline Model 3: as 2 and PA & -1.113 & 4.870 & .824 & 0.179 & .023 \\
\hline Model 4: as 3 and HRT & -0.900 & 4.740 & .854 & 0.181 & .055 \\
\hline \multicolumn{6}{|l|}{ Physical performance } \\
\hline \multicolumn{6}{|l|}{ Vertical jumping height (cm) } \\
\hline Model 1 & -0.080 & 0.081 & .350 & 0.036 & .350 \\
\hline Model 2: adjusted with age & -0.027 & 0.095 & .781 & 0.165 & .231 \\
\hline Model 3: as 2 and PA & -0.022 & 0.093 & .816 & 0.179 & .026 \\
\hline Model 4: as 3 and HRT & -0.031 & 0.097 & .758 & 0.184 & .053 \\
\hline \multicolumn{6}{|l|}{ Hand grip strength $(\mathrm{kg})$} \\
\hline Model 1 & -0.112 & 0.052 & .061 & 0.191 & .061 \\
\hline Model 2: adjusted with age & -0.088 & 0.053 & .127 & 0.269 & .096 \\
\hline Model 3: as 2 and PA & -0.088 & 0.063 & .193 & 0.269 & .049 \\
\hline Model 4: as 3 and HRT & -0.088 & 0.063 & .193 & 0.272 & .123 \\
\hline
\end{tabular}

LTL and SMTL showed a correlation of 0.39 in the study by Ahmad et al. (2012) and 0.71 in the study of Daniali et al. (2013); however, we found no association between LTL and mean and minimum SMTL. We hypothesize that this may be related to the different measurement techniques used (qPCR vs. southern blot) or to the fact that leukocytes and muscle cells are divergent in nature. Leukocytes divide more rapidly than other cell types and they have the lowest mean lifespan (Abramson \& Melton, 2000). Muscle tissue regeneration, in turn, is slower and occurs only through satellite cell proliferation.

As expected, mean SMTL, which mainly consists of telomeres from myonuclei in muscle fibers that have undergone only few cell divisions, was not associated with 
TABLE 4

Associations Between Minimum Skeletal Muscle Telomere Length and Estimates of Total Body and Thigh Composition and Physical Performance in All Subjects $(n=20)$

\begin{tabular}{|c|c|c|c|c|c|}
\hline & \multicolumn{3}{|c|}{ Skeletal muscle minimum telomere length } & \multicolumn{2}{|c|}{ Model* } \\
\hline & $\beta$ & SE & $p$ & $r^{2}$ & $p$ \\
\hline \multicolumn{6}{|l|}{ Body composition } \\
\hline \multicolumn{6}{|l|}{ Body mass index $\left(\mathrm{kg} / \mathrm{m}^{2}\right)$} \\
\hline Model 1 & 0.014 & 0.051 & .795 & 0.009 & .795 \\
\hline Model 2: adjusted with age & -0.017 & 0.043 & .711 & 0.198 & .145 \\
\hline Model 3: as 2 and PA & -0.020 & 0.043 & .658 & 0.208 & .183 \\
\hline Model 4: as 3 and HRT & -0.034 & 0.048 & .497 & 0.257 & .193 \\
\hline \multicolumn{6}{|l|}{ Percentage body fat (\%) } \\
\hline Model 1 & 0.011 & 0.028 & .704 & 0.014 & .704 \\
\hline Model 2: adjusted with age & -0.005 & 0.026 & .863 & 0.189 & .153 \\
\hline Model 3: as 2 and PA & -0.006 & 0.026 & .835 & 0.196 & .171 \\
\hline Model 4: as 3 and HRT & -0.013 & 0.029 & .660 & 0.232 & .177 \\
\hline \multicolumn{6}{|l|}{ Fat-free mass $(\mathrm{kg})$} \\
\hline Model 1 & 0.099 & 0.035 & .020 & 0.274 & .020 \\
\hline Model 2: adjusted with age & 0.078 & 0.029 & .024 & 0.313 & .036 \\
\hline Model 3: as 2 and PA & 0.077 & 0.029 & .028 & 0.316 & .073 \\
\hline Model 4: as 3 and HRT & 0.076 & 0.027 & .021 & 0.336 & .143 \\
\hline \multicolumn{6}{|l|}{ Thigh composition } \\
\hline \multicolumn{6}{|l|}{ Muscle area $\left(\mathrm{m}^{2}\right)$} \\
\hline Model 1 & 4.254 & 1.439 & .016 & 0.417 & .016 \\
\hline Model 2: adjusted with age & 3.831 & 1.272 & .015 & 0.510 & .002 \\
\hline Model 3: as 2 and PA & 3.859 & 1.369 & .020 & 0.511 & .005 \\
\hline Model 4: as 3 and HRT & 3.894 & 1.276 & .014 & 0.541 & .006 \\
\hline \multicolumn{6}{|l|}{ Fat area $\left(\mathrm{m}^{2}\right)$} \\
\hline Model 1 & -0.115 & 0.399 & .780 & 0.007 & .780 \\
\hline Model 2: adjusted with age & -0.389 & 0.375 & .326 & 0.257 & .176 \\
\hline Model 3: as 2 and PA & -0.445 & 0.370 & .260 & 0.280 & .206 \\
\hline Model 4: as 3 and HRT & -0.597 & 0.391 & .161 & 0.356 & .130 \\
\hline \multicolumn{6}{|l|}{ Relative fat (\%) } \\
\hline Model 1 & -0.005 & 0.016 & .760 & 0.007 & .760 \\
\hline Model 2: adjusted with age & -0.014 & 0.019 & .483 & 0.236 & .206 \\
\hline Model 3: as 2 and PA & -0.016 & 0.019 & .418 & 0.255 & .261 \\
\hline Model 4: as 3 and HRT & -0.021 & 0.020 & .338 & 0.313 & .199 \\
\hline \multicolumn{6}{|l|}{ Subcutaneous fat $\left(\mathrm{m}^{2}\right)$} \\
\hline Model 1 & -0.131 & 0.445 & .775 & 0.008 & .775 \\
\hline Model 2: adjusted with age & -0.407 & 0.417 & .355 & 0.252 & .190 \\
\hline Model 3: as 2 and PA & -0.476 & 0.420 & .287 & 0.276 & .219 \\
\hline Model 4: as 3 and HRT & -0.642 & 0.450 & .187 & 0.351 & .135 \\
\hline \multicolumn{6}{|l|}{ Infiltrated fat $\left(\mathrm{m}^{2}\right)$} \\
\hline Model 1 & -0.113 & 3.221 & .973 & 0.000 & .973 \\
\hline Model 2: adjusted with age & -1.529 & 3.477 & .671 & 0.162 & .208 \\
\hline Model 3: as 2 and PA & -1.518 & 3.484 & .673 & 0.214 & .189 \\
\hline Model 4: as 3 and HRT & -1.840 & 3.589 & .620 & 0.248 & .253 \\
\hline \multicolumn{6}{|l|}{ Physical performance } \\
\hline \multicolumn{6}{|l|}{ Vertical jumping height $(\mathrm{cm})$} \\
\hline Model 1 & -0.056 & 0.041 & .204 & 0.117 & .204 \\
\hline Model 2: adjusted with age & -0.037 & 0.042 & .406 & 0.232 & .303 \\
\hline Model 3: as 2 and PA & -0.038 & 0.042 & .379 & 0.241 & .371 \\
\hline Model 4: as 3 and HRT & -0.033 & 0.044 & .467 & 0.252 & .511 \\
\hline \multicolumn{6}{|l|}{ Hand grip strength $(\mathrm{kg})$} \\
\hline Model 1 & 0.013 & 0.032 & .692 & 0.018 & .692 \\
\hline Model 2: adjusted with age & 0.029 & 0.027 & .305 & 0.266 & .017 \\
\hline Model 3: as 2 and PA & 0.031 & 0.031 & .349 & 0.267 & .059 \\
\hline Model 4: as 3 and HRT & 0.031 & 0.032 & .366 & 0.293 & .126 \\
\hline
\end{tabular}

body composition or physical performance. Consequently, we also estimated minimum SMTL, which describes the length of the shortest telomeres present in muscle tissue. Minimum SMTL hypothetically reflects length of telomeres in nuclei of satellite cells and in nuclei newly incorporated into muscle cells (Ponsot et al., 2008). Satellite cell recruitment is an obligatory phenomenon both for skele- tal muscle growth (Hawke \& Garry, 2001) and regeneration. Satellite cell activation increases rapidly in muscles after exercise (Kadi et al., 2004). Their proliferation introduces new myonuclei into muscle cells and contributes to muscle hypertrophy (Kadi \& Thornell, 2000). Hypothetically, longer minimum SMTLs may reflect greater capacity to generate new muscle cells due to inherited or 

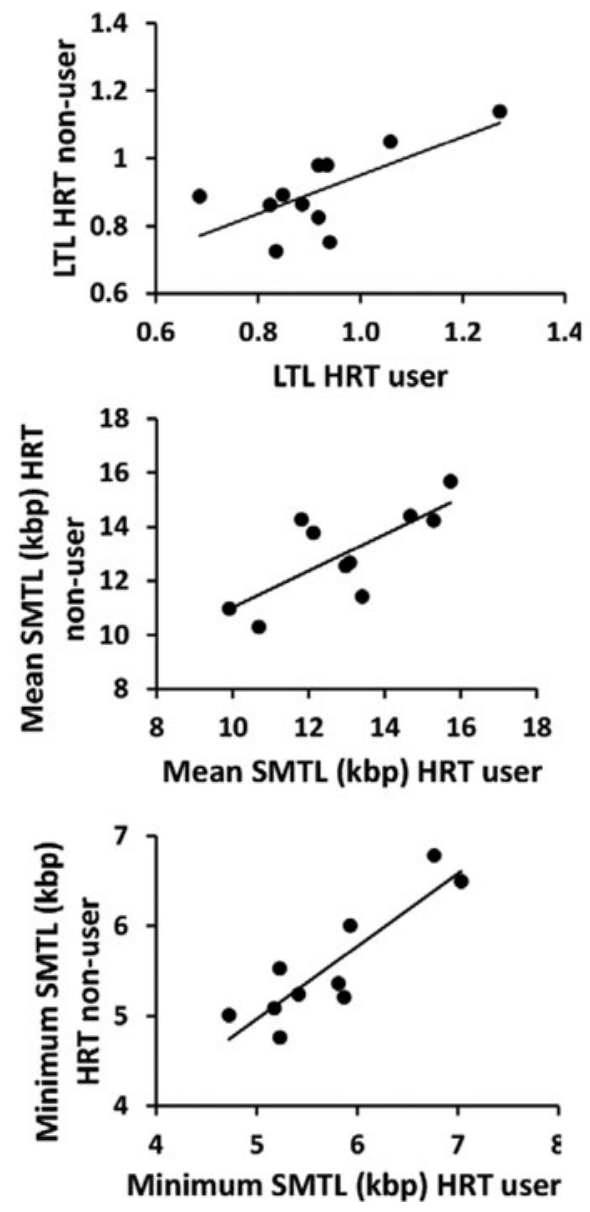

FIGURE 1

Within-pair correlations between HRT users and HRT non-users in (A) relative leukocyte telomere length (LTL, $n=11$ twin pairs), (B) mean skeletal muscle telomere length (SMTL, $n=10$ twin pairs), and (C) minimum skeletal muscle telomere length $(n=10$ twin pairs).

environmental factors. In addition, absence of very short telomeres in muscle cells may indicate better muscle function (Hemann et al., 2001). Our results, which showed that longer minimum SMTLs were associated with higher FFM and greater muscle area in the thigh, support the hypothesis that minimum SMTL is a specific biomarker of skeletal muscle mass. The association observed between higher minimum SMTLs and increasing muscle mass is supported by Kadi et al. (2008), who also found that minimum SMTLs tended to be longer in subjects with higher muscle mass. Whether length of the minimum SMTLs limits FFM at older age, or whether telomere shortening and decrease in FFM are simultaneously age-related, possibly genetically regulated (Arden \& Spector, 1997) processes, needs more research.

Overall, cross-sectional studies offer little evidence about associations between LTL and physical performance (Gardner et al., 2013). Conflicting findings have been
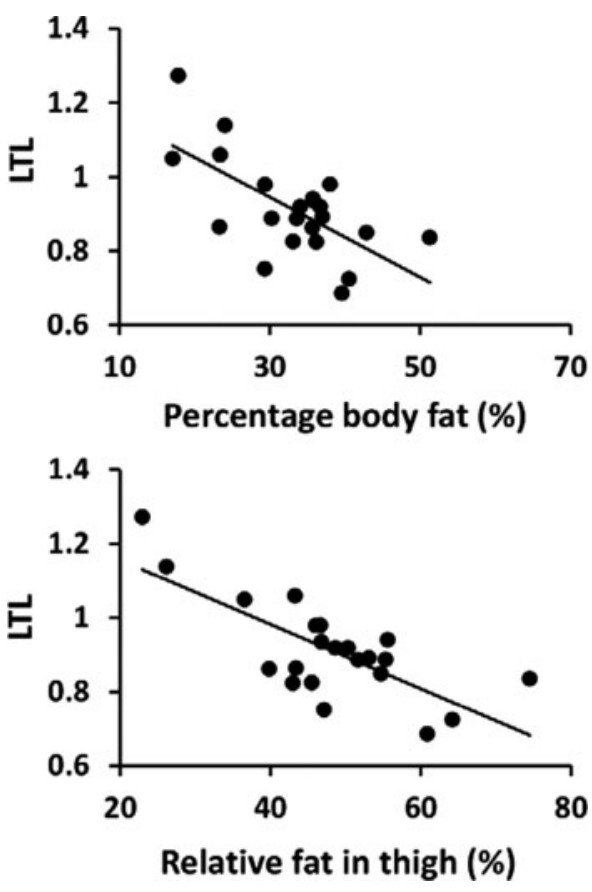

FIGURE 2

Association between relative leukocyte telomere length (LTL) and (A) percentage body fat (\%), and (B) relative proportion of fat in thigh (\%) in all subjects ( $n=22$ individuals).
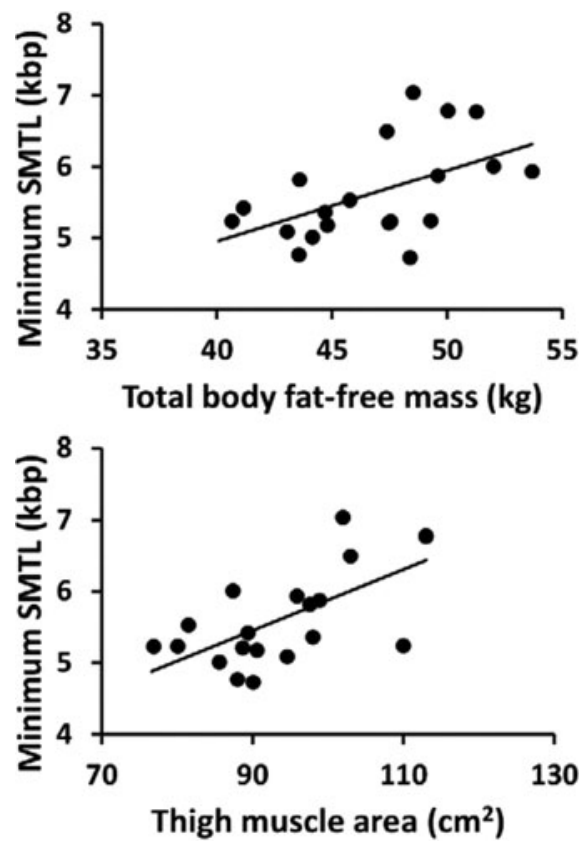

\section{FIGURE 3}

Association between minimum skeletal muscle telomere length (SMTL) and (A) total body fat-free mass $(\mathrm{kg})$ and $(B)$ thigh muscle cross-sectional area $\left(\mathrm{cm}^{2}\right)$ in all subjects $(n=20$ individuals). 
reported on the association between LTL and walking speed (Gardner et al., 2013; Harris et al., 2006; Lee et al., 2013) and LTL and grip strength (Der et al., 2012; Harris et al., 2006). We have previously shown that LTL predicts the decline in PA and walking ability in older twin sisters (Sillanpaa et al., 2016). Data on SMTL is even scarcer, as it is limited to studies that have used muscle biopsies. High-intensity training causes an increased demand on skeletal muscle to repair, regenerate, and remodel muscle tissue, which in turn shortens minimum telomere length in skeletal muscle. Rae et al. (2010) reported an inverse relationship between minimum telomere length and long-term exposure to endurance exercise. Kadi et al. (2008) observed an inverse correlation between minimum SMTL and performance in power lifting. Our results suggest no or only weak associations between telomeres and physical performance. LTL approached significance as an independent predictor of hand grip strength $(p=.060)$, which is a performance-based measurement known to be highly related to aging, overall health, and mortality (Rantanen et al., 2003).

In conclusion, findings from our genetically controlled twin data suggest that long-term use of HRT has no or at most very small effects on LTL and SMTL in postmenopausal women. It is possible that natural aging, associated with attenuation in sex hormones as well as telomere attrition, are regulated through genetic factors. Previous findings that HRT use is associated with longer LTLs (Lee et al., 2005) are possibly explained by selection bias or confounding effects of HRT. HRT users seem to be more prone to adopt a healthier lifestyle (Lee et al., 2005), while the use of HRT might result in more favorable body composition and better physical performance. Based on the findings of the present study and earlier investigations (Lee et al., 2011; Njajou et al., 2009; Nordfjall, Eliasson, Stegmayr, Lundin et al., 2008), it is likely that a healthier body composition is associated with longer telomere lengths and that the associations may differ with respect to the cell types in which telomeres are determined.

\section{Acknowledgments}

The authors acknowledge Dr Iiris Hovatta and Ms Laura Kananen for their help in carrying out the telomere length analysis by qPCR. We also acknowledge support from the EC FP7 Collaborative Project MYOAGE (GA-223576). The Gerontology Research Center is a joint effort between the University of Jyväskylä and the University of Tampere. The SAWEs study was funded by the Academy of Finland and the Finnish Ministry of Culture and Education (V.K.: grant numbers 251316 and 89/672/2008). E.S. is supported by a post doc research grant from the Academy of Finland (grant number 260001). J.K. has been supported by the Academy of Finland (grants 265240, 263278).

\section{References}

Abramson, N., \& Melton, B. (2000). Leukocytosis: Basics of clinical assessment. American Family Physician, 62, 20532060.

Ahmad, S., Heraclides, A., Sun, Q., Elgzyri, T., Ronn, T., Ling, C., ... Hansson, O. (2012). Telomere length in blood and skeletal muscle in relation to measures of glycaemia and insulinaemia. Diabetic Medicine, 29, e377-e381.

Allsopp, R. C., Vaziri, H., Patterson, C., Goldstein, S., Younglai, E. V., Futcher, A. B., ... Harley, C. B. (1992). Telomere length predicts replicative capacity of human fibroblasts. Proceedings of the National Academy of Sciences, 89, 1011410118.

Alway, S. E., Myers, M. J., \& Mohamed, J. S. (2014). Regulation of satellite cell function in sarcopenia. Frontiers in Aging Neuroscience, 6, 246.

Arden, N. K., \& Spector, T. D. (1997). Genetic influences on muscle strength, lean body mass, and bone mineral density: A twin study. Journal of Bone and Mineral Research, 12, 2076-2081.

Aubert, G., Baerlocher, G. M., Vulto, I., Poon, S. S., \& Lansdorp, P. M. (2012). Collapse of telomere homeostasis in hematopoietic cells caused by heterozygous mutations in telomerase genes. PLoS Genetics, 8, e1002696.

Aviv, A. (2002). Chronology versus biology: Telomeres, essential hypertension, and vascular aging. Hypertension, 40, 229-232.

Aviv, A., Shay, J., Christensen, K., \& Wright, W. (2005). The longevity gender gap: Are telomeres the explanation? Science of Aging Knowledge Environment, 23, pe16.

Aviv, A., Valdes, A., Gardner, J. P., Swaminathan, R., Kimura, M., \& Spector, T. D. (2006). Menopause modifies the association of leukocyte telomere length with insulin resistance and inflammation. The Journal of Clinical Endocrinology and Metabolism, 91, 635-640.

Aydos, S. E., Elhan, A. H., \& Tukun, A. (2005). Is telomere length one of the determinants of reproductive life span? Archives of Gynecology and Obstetrics, 272, 113-116.

Benetos, A., Okuda, K., Lajemi, M., Kimura, M., Thomas, F., Skurnick, J., ... Aviv, A. (2001). Telomere length as an indicator of biological aging: The gender effect and relation with pulse pressure and pulse wave velocity. Hypertension, 37, 381-385.

Blasco, M. A. (2005). Telomeres and human disease: Ageing, cancer and beyond. Nature Reviews.Genetics, 6, 611-622.

Bodnar, A. G., Ouellette, M., Frolkis, M., Holt, S. E., Chiu, C. P., Morin, G. B., ... Wright, W. E. (1998). Extension of life-span by introduction of telomerase into normal human cells. Science, 279, 349-352.

Bosco, C., Luhtanen, P., \& Komi, P. V. (1983). A simple method for measurement of mechanical power in jumping. European Journal of Applied Physiology and Occupational Physiology, 50, 273-282.

Broer, L., Codd, V., Nyholt, D. R., Deelen, J., Mangino, M., Willemsen, G., ... Boomsma, D. I. (2013). Metaanalysis of telomere length in 19,713 subjects reveals high 
heritability, stronger maternal inheritance and a paternal age effect. European Journal of Human Genetics, 21, 1163-1168.

Cawthon, R. M., Smith, K. R., O’Brien, E., Sivatchenko, A., \& Kerber, R. A. (2003). Association between telomere length in blood and mortality in people aged 60 years or older. Lancet, 361, 393-395.

Chen, W., Kimura, M., Kim, S., Cao, X., Srinivasan, S. R., Berenson, G. S., ... Aviv, A. (2011). Longitudinal versus cross-sectional evaluations of leukocyte telomere length dynamics: Age-dependent telomere shortening is the rule. The Journals of Gerontology Series A: Biological Sciences and Medical Sciences, 66, 312-319.

Cherkas, L. F., Hunkin, J. L., Kato, B. S., Richards, J. B., Gardner, J. P., Surdulescu, G. L., ... Aviv, A. (2008). The association between physical activity in leisure time and leukocyte telomere length. Archives of Internal Medicine, $168,154-158$.

Dalgard, C., Benetos, A., Verhulst, S., Labat, C., Kark, J. D., Christensen, K., ... Aviv, A. (2015). Leukocyte telomere length dynamics in women and men: Menopause vs age effects. International Journal of Epidemiology, 44, 1688-1695.

Daniali, L., Benetos, A., Susser, E., Kark, J. D., Labat, C., Kimura, M., ... Aviv, A. (2013). Telomeres shorten at equivalent rates in somatic tissues of adults. Nature Communications, 4, 1597.

de Lange, T. (2006). Lasker laurels for telomerase. Cell, 126, 1017-1020.

Decary, S., Mouly, V., Hamida, C. B., Sautet, A., Barbet, J. P., \& Butler-Browne, G. S. (1997). Replicative potential and telomere length in human skeletal muscle: Implications for satellite cell-mediated gene therapy. Human Gene Therapy, $8,1429-1438$.

Demissie, S., Levy, D., Benjamin, E. J., Cupples, L. A., Gardner, J. P., Herbert, A., ... Aviv, A. (2006). Insulin resistance, oxidative stress, hypertension, and leukocyte telomere length in men from the framingham heart study. Aging Cell, 5, 325-330.

Der, G., Batty, G. D., Benzeval, M., Deary, I. J., Green, M. J., McGlynn, L., ... Shiels, P. G. (2012). Is telomere length a biomarker for aging: Cross-sectional evidence from the west of scotland? PloS One, 7, e45166.

Du, M., Prescott, J., Kraft, P., Han, J., Giovannucci, E., Hankinson, S. E., \& De Vivo, I. (2012). Physical activity, sedentary behavior, and leukocyte telomere length in women. American Journal of Epidemiology, 175, 414-422.

Eerola, J., Kananen, L., Manninen, K., Hellstrom, O., Tienari, P. J., \& Hovatta, I. (2010). No evidence for shorter leukocyte telomere length in parkinson's disease patients. The Journals of Gerontology.Series A, Biological Sciences and Medical Sciences, 65, 1181-1184.

Epel, E. S., Blackburn, E. H., Lin, J., Dhabhar, F. S., Adler, N. E., Morrow, J. D., \& Cawthon, R. M. (2004). Accelerated telomere shortening in response to life stress. Proceedings of the National Academy of Sciences of the United States of America, 101, 17312-17315.
Gardner, M. P., Martin-Ruiz, C., Cooper, R., Hardy, R., Sayer, A. A., Cooper, C., ... Halcyon Study Team. (2013). Telomere length and physical performance at older ages: An individual participant meta-analysis. PloS One, 8, e69526.

Grasselli, A., Nanni, S., Colussi, C., Aiello, A., Benvenuti, V., Ragone, G., ... Farsetti, A. (2008). Estrogen receptor-alpha and endothelial nitric oxide synthase nuclear complex regulates transcription of human telomerase. Circulation Research, 103, 34-42.

Gray, K. E., Schiff, M. A., Fitzpatrick, A. L., Kimura, M., Aviv, A., \& Starr, J. R. (2014). Leukocyte telomere length and age at menopause. Epidemiology, 25, 139-146.

Harris, S. E., Deary, I. J., MacIntyre, A., Lamb, K. J., Radhakrishnan, K., Starr, J. M., ... Shiels, P. G. (2006). The association between telomere length, physical health, cognitive ageing, and mortality in non-demented older people. Neuroscience Letters, 406, 260-264.

Hawke, T. J., \& Garry, D. J. (2001). Myogenic satellite cells: Physiology to molecular biology. Journal of Applied Physiology, 91, 534-551.

Haycock, P. C., Heydon, E. E., Kaptoge, S., Butterworth, A. S., Thompson, A., \& Willeit, P. (2014). Leucocyte telomere length and risk of cardiovascular disease: Systematic review and meta-analysis. BMJ, 349, g4227.

Hemann, M. T., Strong, M. A., Hao, L. Y., \& Greider, C. W. (2001). The shortest telomere, not average telomere length, is critical for cell viability and chromosome stability. Cell, 107, 67-77.

Hjelmborg, J. B., Dalgard, C., Moller, S., Steenstrup, T., Kimura, M., Christensen, K., ... Aviv, A. (2015). The heritability of leucocyte telomere length dynamics. Journal of Medical Genetics, 52, 297-302.

Huzen, J., de Boer, R. A., van Veldhuisen, D. J., van Gilst, W. H., \& van der Harst, P. (2010). The emerging role of telomere biology in cardiovascular disease. Frontiers in Bioscience, 15, 35-45.

Kadi, F., Ponsot, E., Piehl-Aulin, K., Mackey, A., Kjaer, M., Oskarsson, E., \& Holm, L. (2008). The effects of regular strength training on telomere length in human skeletal muscle. Medicine and Science in Sports and Exercise, 40, 8287.

Kadi, F., Schjerling, P., Andersen, L. L., Charifi, N., Madsen, J. L., Christensen, L. R., \& Andersen, J. L. (2004). The effects of heavy resistance training and detraining on satellite cells in human skeletal muscles. The Journal of Physiology, 558, 1005-1012.

Kadi, F., \& Thornell, L. E. (2000). Concomitant increases in myonuclear and satellite cell content in female trapezius muscle following strength training. Histochemistry and Cell Biology, 113, 99-103.

Kananen, L., Surakka, I., Pirkola, S., Suvisaari, J., Lonnqvist, J., Peltonen, L., ... Hovatta, I. (2010). Childhood adversities are associated with shorter telomere length at adult age both in individuals with an anxiety disorder and controls. PloS One, 5, e10826.

Kangas, R., Pollanen, E., Rippo, M. R., Lanzarini, C., Prattichizzo, F., Niskala, P., ... Kovanen, V. (2014). Circulating miR-21, miR-146a and fas ligand respond to 
postmenopausal estrogen-based hormone replacement therapy - a study with monozygotic twin pairs. Mechanisms of Ageing and Development, 143-144, 1-8.

Kaprio, J., \& Koskenvuo, M. (2002). Genetic and environmental factors in complex diseases: The older finnish twin cohort. Twin Research, 5, 358-365.

Kyo, S., Takakura, M., Kanaya, T., Zhuo, W., Fujimoto, K., Nishio, Y., ... Inoue, M. (1999). Estrogen activates telomerase. Cancer Research, 59, 5917-5921.

LaRocca, T. J., Seals, D. R., \& Pierce, G. L. (2010). Leukocyte telomere length is preserved with aging in endurance exercise-trained adults and related to maximal aerobic capacity. Mechanisms of Ageing and Development, 131, 165-167.

Lee, D. C., Im, J. A., Kim, J. H., Lee, H. R., \& Shim, J. Y. (2005). Effect of long-term hormone therapy on telomere length in postmenopausal women. Yonsei Medical Journal, 46, 471479.

Lee, J. Y., Bang, H. W., Ko, J. H., Kim, J. H., \& Lee, D. C. (2013). Leukocyte telomere length is independently associated with gait speed in elderly women. Maturitas, 75, 165-169.

Lee, M., Martin, H., Firpo, M. A., \& Demerath, E. W. (2011). Inverse association between adiposity and telomere length: The fels longitudinal study. American Journal of Human Biology, 23, 100-106.

Lin, J., Kroenke, C. H., Epel, E., Kenna, H. A., Wolkowitz, O. M., Blackburn, E., \& Rasgon, N. L. (2011). Greater endogenous estrogen exposure is associated with longer telomeres in postmenopausal women at risk for cognitive decline. Brain Research, 1379, 224-231.

Misiti, S., Nanni, S., Fontemaggi, G., Cong, Y. S., Wen, J., Hirte, H. W., ... Farsetti, A. (2000). Induction of hTERT expression and telomerase activity by estrogens in human ovary epithelium cells. Molecular and Cellular Biology, 20, 37643771.

Njajou, O. T., Cawthon, R. M., Blackburn, E. H., Harris, T. B., Li, R., Sanders, J. L., ... Hsueh, W. C. (2012). Shorter telomeres are associated with obesity and weight gain in the elderly. International Journal of Obesity (London), 36, 11761179.

Njajou, O. T., Hsueh, W. C., Blackburn, E. H., Newman, A. B., Wu, S. H., Li, R., ... Health ABC study. (2009). Association between telomere length, specific causes of death, and years of healthy life in health, aging, and body composition: A population-based cohort study. The Journals of Gerontology.Series A, Biological Sciences and Medical Sciences, 64, 860-864.

Nordfjall, K., Eliasson, M., Stegmayr, B., Lundin, S., Roos, G., \& Nilsson, P. M. (2008). Increased abdominal obesity, adverse psychosocial factors and shorter telomere length in subjects reporting early ageing; the MONICA northern Sweden study. Scandinavian Journal of Public Health, 36, 744-752.

Nordfjall, K., Eliasson, M., Stegmayr, B., Melander, O., Nilsson, P., \& Roos, G. (2008). Telomere length is associated with obesity parameters but with a gender difference. Obesity (Silver Spring), 16, 2682-2689.
Oeseburg, H., de Boer, R. A., van Gilst, W. H., \& van der Harst, P. (2010). Telomere biology in healthy aging and disease. European Journal of Physiology, 459, 259-268.

Pollanen, E., Ronkainen, P. H., Suominen, H., Takala, T., Koskinen, S., Puolakka, J., ... Kovanen, V. (2007). Muscular transcriptome in postmenopausal women with or without hormone replacement. Rejuvenation Research, 10, 485-500.

Ponsot, E., \& Kadi, F. (2008). Signal modelization for improved precision of assessment of minimum and mean telomere lengths. Electrophoresis, 29, 542-544.

Ponsot, E., Echaniz-Laguna, A., Delis, A. M., \& Kadi, F. (2012). Telomere length and regulatory proteins in human skeletal muscle with and without ongoing regenerative cycles. Experimental Physiology, 97, 774-784.

Ponsot, E., Lexell, J., \& Kadi, F. (2008). Skeletal muscle telomere length is not impaired in healthy physically active old women and men. Muscle \& Nerve, 37, 467-472.

Rae, D. E., Vignaud, A., Butler-Browne, G. S., Thornell, L. E., Sinclair-Smith, C., Derman, E. W., ... Collins, M. (2010). Skeletal muscle telomere length in healthy, experienced, endurance runners. European Journal of Applied Physiology, 109, 323-330.

Rahimian, R., Chan, L., Goel, A., Poburko, D., \& van Breemen, C. (2004). Estrogen modulation of endothelium-derived relaxing factors by human endothelial cells. Biochemical and Biophysical Research Communications, 322, 373-379.

Rantanen, T., Volpato, S., Ferrucci, L., Heikkinen, E., Fried, L. P., \& Guralnik, J. M. (2003). Handgrip strength and cause-specific and total mortality in older disabled women: Exploring the mechanism. Journal of the American Geriatrics Society, 51, 636-641.

Ronkainen, P. H., Kovanen, V., Alen, M., Pollanen, E., Palonen, E. M., Ankarberg-Lindgren, C., ... Sipila, S. (2009). Postmenopausal hormone replacement therapy modifies skeletal muscle composition and function: A study with monozygotic twin pairs. Journal of Applied Physiology, 107, 25-33.

Ronkainen, P. H., Pollanen, E., Alen, M., Pitkanen, R., Puolakka, J., Kujala, U. M., ... Kovanen, V. (2010). Global gene expression profiles in skeletal muscle of monozygotic female twins discordant for hormone replacement therapy. Aging Cell, 9, 1098-1110.

Sillanpaa, E., Tormakangas, T., Rantanen, T., Kaprio, J., \& Sipila, S. (2016). Does telomere length predict decline in physical functioning in older twin sisters during an 11-year follow-up? Age, 38, 34.

Sipila, S., Taaffe, D. R., Cheng, S., Puolakka, J., Toivanen, J., \& Suominen, H. (2001). Effects of hormone replacement therapy and high-impact physical exercise on skeletal muscle in post-menopausal women: A randomized placebocontrolled study. Clinical Science, 101, 147-157.

Song, J. Y., Kim, M. J., Jo, H. H., Hwang, S. J., Chae, B., Chung, J. E., ... Kim, M. R. (2009). Antioxidant effect of estrogen on bovine aortic endothelial cells. Journal of Steroid Biochemistry and Molecular Biology, 117, 74-80.

Valdes, A. M., Andrew, T., Gardner, J. P., Kimura, M., Oelsner, E., Cherkas, L. F., ... Spector, T. D. (2005). Obesity, cigarette 
smoking, and telomere length in women. Lancet, 366, 662664.

Vasa, M., Breitschopf, K., Zeiher, A. M., \& Dimmeler, S. (2000). Nitric oxide activates telomerase and delays endothelial cell senescence. Circulation Research, 87, 540-542.

Wernig, A., Schafer, R., Knauf, U., Mundegar, R. R., Zweyer, M., Hogemeier, O., ... Zimmermann, S. (2005). On the regenerative capacity of human skeletal muscle. Artificial Organs, 29, 192-198.
Wong, C. M., Yung, L. M., Leung, F. P., Tsang, S. Y., Au, C. L., Chen, Z. Y., ... Huang, Y. (2008). Raloxifene protects endothelial cell function against oxidative stress. British Journal of Pharmacology, 155, 326-334.

Zeichner, S. L., Palumbo, P., Feng, Y., Xiao, X., Gee, D., Sleasman, J., ... Dimitrov, D. (1999). Rapid telomere shortening in children. Blood, 93, 2824-2830. 